\section{Early determinants of attention and hyperactivity problems in adolescents: the 11-year follow-up of the 1993 Pelotas (Brazil) birth cohort study}

\author{
Determinantes precoces de problemas de atenção e \\ hiperatividade na adolescência: a visita de 11 anos \\ da coorte de nascimentos de Pelotas, Rio Grande \\ do Sul, Brasil, 1993
}

\author{
${ }^{1}$ Faculdade de Medicina, \\ Universidade Federal do Rio \\ Grande do Sul, Porto Alegre, \\ Brasil. \\ 2 Programa de Pós-graduação \\ em Epidemiologia, \\ Universidade Federal de \\ Pelotas, Pelotas, Brasil. \\ 3 Programa de Pós-graduação \\ em Saúde e Comportamento, \\ Universidade Católica de \\ Pelotas, Pelotas, Brasil. \\ 4 Programa de Pós-graduação \\ em Educação Física, \\ Universidade Federal de \\ Pelotas, Pelotas, Brasil. \\ 5 Faculdade de Nutrição, \\ Universidade Federal de \\ Pelotas, Pelotas, Brasil. \\ ${ }^{6}$ Hospital das Clínicas de \\ Porto Alegre, Universidade \\ Federal do Rio Grande do Sul \\ Porto Alegre, Brasil. \\ Correspondence \\ L. Anselmi \\ Faculdade de Medicina, \\ Universidade Federal do \\ Rio Grande do Sul. \\ Rua Dr. Pedro Armando Gatti \\ 155, Rio Grande, RS 96216 \\ 080, Brasil. \\ luanselmi@terra.com.br
}

\begin{abstract}
The aim of this study was to assess early determinants of attention and hyperactivity problems in adolescents. In 1993, all hospital births in the city of Pelotas, Rio Grande do Sul State, Brazil, were monitored and mothers were interviewed $(N=5,249)$. At 11 years of age, 4,423 mothers answered the Strengths and Difficulties Questionnaire (SDQ) in order to evaluate attention and hyperactivity problems in the adolescents. Crude and adjusted prevalence ratios were calculated using Poisson regression. Prevalence of attention and hyperactivity problems was 19.9\%. Factors associated with the outcome in the adjusted analysis were: male gender, low family income, smoking during pregnancy, minor psychiatric disorders in the mother, and history of child's behavioral/emotional problems at four years of age. Early life events impacted attention and hyperactivity problems in adolescence. Risk factors for attention and hyperactivity problems found in this study were similar to those reported in other cultures.
\end{abstract}

Attention Deficit Disorder with Hyperactivity; Adolescent; Cohort Studies
Luciana Anselmi 1,2 Ana M. B. Menezes 2 Fernando C. Barros 3 Pedro C. Hallal 4

Cora Luiza Araújo 2,5 Marlos R. Domingues 2 Luis A. Rohde 6

\section{Introduction}

Attention deficit hyperactivity disorder (ADHD) is characterized by persistent and severe symptoms of inattention, hyperactivity, and impulsiveness 1 . With early onset, ADHD is one of the main reasons for consulting pediatric mental health services 2 and is among the most frequent mental disorders in childhood and adolescence ${ }^{3}$. It can also have a negative impact on scholastic performance and family and peer relations 3 .

Although ADHD is a genetically determined condition - approximately $75 \%$ of the variability in inattention and hyperactivity in population samples is explained by genetic factors 3 -, various biological and environmental risk factors also contribute to its etiology, probably exerting their effect through interaction with genetic factors 4 . Epidemiological studies 5,6 have shown that ADHD is more common in children from low-income families. Among the demographic factors, gender is the principal predictor, and the disorder is three times more frequent in boys than in girls according to community studies 7. Meanwhile, the findings for ethnic or racial differences in ADHD are less consistent 3,8 .

Studies have reported a long-term effect of maternal smoking during pregnancy 9,10, although the causal link between nicotine and ADHD has not been well-established 11. Maternal alcohol consumption, pregnancy and birth 
complications, prematurity, and low birth weight are other possible risk factors 3,4

Children and adolescents with ADHD frequently present comorbidity with other mental disorders and below-average intelligence quotient (IQ) ${ }^{3}$. In addition, youngsters whose parents present a mental disorder have a higher likelihood of developing ADHD 3,12.

The current study aimed to investigate risk factors present at birth and at four years of age for later problems with attention and hyperactivity in adolescents from a birth cohort in the city of Pelotas, Rio Grande do Sul State, Brazil.

\section{Methods}

All hospital births in the city of Pelotas in 1993 were monitored. The mothers answered a questionnaire and the newborns were weighed and measured. Sub-samples of these individuals were visited at 1,3 , and 6 months and $1,4,6$, and 9 years of age. In 2004-2005, all the cohort members were searched for a new follow-up, and 4,452 mothers (representing $87.5 \%$ of the original cohort) were interviewed. This article draws on data collected at the perinatal visit and the 4 and 11-year followup visits. A detailed description of the follow-up visits and study variables can be found in the methods article in this Supplement 13.

The following variables were used from the perinatal study $(\mathrm{N}=5,249)$ : family income (measured as times the prevailing minimum wage), child's sex, gestational age (date of last menstrual period and Dubowitz method performed on the first day of life in the newborns 14), birth weight (newborns were weighed at birth with pediatric scales, Filizola brand, São Paulo, Brazil, accurate to $10 \mathrm{~g}$, calibrated weekly with standard weights), intrauterine growth restriction (Kramer criterion), maternal complications during pregnancy (mother's hospitalization during the pregnancy), smoking during pregnancy (mothers answered "yes/no" as to whether they had smoked during the pregnancy), alcohol consumption during pregnancy (mothers answered "yes/no" as to whether they had consumed alcohol during the pregnancy), and perinatal complications (infant's admission to the neonatal ICU).

When the children were four years of age, the families were visited to assess the mental health of a subsample of children from the cohort ( $\mathrm{n}=$ 634 ), or half of the sample visited at 12 months. This follow-up used the following variables: behavioral and emotional problems, assessed with the Child Behavior Checklist (CBCL) 15, and IQ, assessed with the Wechsler Preschool and Primary Scale of Intelligence (WPPSI) 16 in the children, plus minor mental disorders presented by the mothers, assessed by the Self-Report Questionnaire (SRQ-20) 17. The "IQ" variable was categorized in three groups. The first group included children with high and medium-high IQ, the second included medium and medium-low IQ, and the third group included borderline children and those with mental disabilities. The variable "behavioral and emotional problems" was dichotomized, and the children with scores $\geq 2$ standarddeviations (SD) were considered positive for this outcome. The variable "maternal mental disorder" was dichotomized, and mothers with scores $\geq 8$ were considered "positive" 17 .

From the 2004-2005 follow-up ( $\mathrm{N}=4,452)$, when the cohort members were an average of 11.3 years old $(\mathrm{SD}=0.3)$, the study used the variables self-reported skin color (black and brown were combined into one group) and attention and hyperactivity problems. To evaluate attention and hyperactivity problems, 4,423 mothers or guardians answered the Strengths and Difficulties Questionnaire (SDQ) 18. The SDQ is a screening questionnaire that assesses mental problems in children and adolescents in the six months prior to the interview. It consists of 25 questions divided into five sub-scales with five questions each, generating scores for emotional symptoms, conduct problems, inattention, hyperactivity, problems with peer relations, and pro-social behavior problems. For the outcome, the study used a sub-scale of attention and hyperactivity problems from the parents' version of SDQ, since parents tend to report symptoms of inattention and hyperactivity better than the adolescents themselves 19. The SDQ was developed by Goodman 18 and validated in Brazil by Fleitlich-Bilyk 20.

The WPPSI 16 was used to assess the children's IQ at four years. We used an abbreviated version consisting of two verbal sub-tests (comprehension and arithmetic) and two executive sub-tests (completing figures and building with blocks). Developed by Wechsler, this scale was adapted in Argentina 16.

The CBCL was used to assess behavioral and emotional problems. It was developed by Achenbach 15 and adapted in Brazil by Bordin et al. 21 .

The SRQ-20 assessed the presence of common (non-psychotic) mental disorders in mothers, especially depression and anxiety in the previous month. This scale was developed by Harding et al. 22 and validated in Brazil by Mari \&Williams 17 .

The statistical analyses were performed with Stata 9.0 (Stata Corp., College Station, USA) and SPSS 13.0 (SPSS Inc., Chicago, USA). The outcome was the dichotomized score from the SDQ 
attention and hyperactivity scale. The cutoff for the scale (score $\geq 8$ ) was chosen based on a pilot study, when a subsample of cohort participants ( $n=280$ ) was extensively assessed using a semistructured interview, Development and Well-Being Assessment (DAWBA) 20. We used ROC curve analyses with ADHD diagnosis according to the Diagnostic and Statistical Manual of Mental Disorders (DSM-IV) ${ }^{1}$ as the parameter for determining the cutoff. The area below the curve for the SDQ attention and hyperactivity scale was 0.80 (95\% confidence level - 95\%CI: 0.71; 0.88), when compared to the ADHD diagnosis, was based on the DAWBA for this cutoff.

In the descriptive analysis, prevalence rates were obtained for attention and hyperactivity problems with $95 \% \mathrm{CI}$, using chi-square tests for heterogeneity or linear trend, seeking to compare the proportions of attention and hyperactivity problems among the groups of independent variables. Both the crude and adjusted analyses used Poisson regression with robust adjustment of variance 23 .

The adjusted analysis followed a hierarchical conceptual model 24 according to the levels of determination of hyperactivity problems and with temporal data collection questions. At the first level, the socioeconomic and demographic variables were entered: family income and the adolescent's gender and skin color. The pregnancy variables collected at the perinatal follow-up were entered at the second level: smoking and alcohol consumption and maternal complications during pregnancy. The perinatal variables were entered at the third level: prematurity, intrauterine growth restriction, and perinatal complications. The fourth level included the psychological variables collected in the fourth year: behavioral and emotional problems and child's IQ, plus mother's mental disorder. The next step included the variables with $\mathrm{p}<0.20$ in the previous stage in the analytical model. A second adjusted analysis was performed, with the aim of verifying the adequacy of the chosen cutoff point for SDQ based on DAWBA. A linear regression was performed with the same hierarchical strategy of including variables in the model, but using the outcome (the SDQ parents' score for attention and hyperactivity problem in the children) continuously.

The project was approved by the Institutional Review Board of the School of Medicine, Federal University in Pelotas (UFPel). Parents or guardians signed a free and informed consent form authorizing their own participation and that of the children in the study. Mothers that requested psychological care were referred to the adolescent mental health outpatient clinic at UFPel.

\section{Results}

The prevalence of attention and hyperactivity problems was $19.9 \%$ (95\%CI: $18.7 ; 21.1)$ at 11 years of age. Table 1 shows the prevalence and confidence intervals for attention and hyperactivity problems according to the variables collected at birth. Male gender, black/brown skin color, low family income, smoking, and pregnancy complications were associated with attention and hyperactivity problems at 11 years. No statistically significant associations $(p \leq 0.05)$ were found with the other perinatal variables.

Table 2 shows the prevalence of attention and hyperactivity problems according to variables collected at the follow-up at four years of age. Low IQ and behavioral/emotional problems in the child and maternal psychiatric disorders were associated with attention and hyperactivity problems at 11 years.

Table 3 shows the crude and adjusted analysis using Poisson regression. The effect of family income and gender was not modified after adjusting for confounding factors. Meanwhile, skin color lost its association with attention and hyperactivity problems after controlling for socioeconomic and demographic variables. Maternal smoking during pregnancy was the only factor that remained statistically significant after adjusting for the other socioeconomic, demographic, and gestational variables that entered the second level of the equation. In this adjusted model, the child's behavioral/emotional problems and mother's psychiatric disorder remained associated with attention and hyperactivity problems at 11 years. On the other hand the child's IQ at four years did not remain associated after adjustment.

In the second adjusted linear regression analysis with the continuous outcome, the final model was very similar to that shown in Table 3 . The only change was in IQ, which remained associated with attention and hyperactivity problems, while behavioral/emotional problems did not remain associated.

\section{Discussion}

Among the environmental, biological, and psychological variables that comprised the analytical model, five were associated with attention and hyperactivity problems in early adolescence: low family income, adolescent's gender, smoking during pregnancy, behavioral/emotional problems, and maternal mental disorder.

The increased risk of developing attention and hyperactivity problems in adolescents from low- 
Prevalence of attention and hyperactivity problems in adolescence according to variables collected at birth. 1993 Pelotas (Brazil) birth cohort study, 2004-2005 follow-up.

\begin{tabular}{|c|c|c|}
\hline Variables & $\%(95 \% \mathrm{Cl})$ & p-value \\
\hline Gender & & $<0.001$ \\
\hline Female & $16.0(14.4-17.5)$ & \\
\hline Male & $24.0(22.2-25.7)$ & \\
\hline Skin color & & 0.017 \\
\hline White & $18.7(17.3-20.1)$ & \\
\hline Black/Brown & $21.7(19.4-24.0)$ & \\
\hline Family income (times minimum wage) & & $<0.001$ \\
\hline$>10$ & $9.0(5.8-12.2)$ & \\
\hline $6.1-10$ & $15.5(11.6-19.4)$ & \\
\hline $3.1-6$ & $17.5(15.2-19.8)$ & \\
\hline $1.1-3$ & $22.6(20.7-24.5)$ & \\
\hline$\leq 1$ & $22.6(19.7-25.5)$ & \\
\hline Smoking during pregnancy & & $<0.001$ \\
\hline No & $17.8(16.4-19.2)$ & \\
\hline Yes & $24.1(21.9-26.3)$ & \\
\hline Alcohol during pregnancy & & 0.169 \\
\hline No & $19.7(18.5-20.9)$ & \\
\hline Yes & $23.5(17.9-29.0)$ & \\
\hline Birth weight & & 0.366 \\
\hline Adequate $(\geq 2,500 \mathrm{~g})$ & $19.7(18.5-20.9)$ & \\
\hline Low $(<2,500 \mathrm{~g})$ & $21.6(17.5-25.7)$ & \\
\hline Gestational age (weeks) & & 0.067 \\
\hline$\geq 37$ & $19.5(18.2-20.7)$ & \\
\hline$<37$ (premature) & $23.0(19.2-26.9)$ & \\
\hline Intrauterine growth restriction & & 0.131 \\
\hline No & $19.2(17.8-20.6)$ & \\
\hline Yes & $21.4(18.8-24.0)$ & \\
\hline Pregnancy complications & & 0.025 \\
\hline No hospitalization & $19.5(18.8-20.7)$ & \\
\hline With hospitalization & $24.4(20.0-28.9)$ & \\
\hline Perinatal complications & & 0.112 \\
\hline No neonatal ICU admission & $19.7(18.6-20.9)$ & \\
\hline Neonatal ICU admission & $25.9(17.5-34.3)$ & \\
\hline
\end{tabular}

95\% Cl: 95\% confidence interval.

income families may be related to their increased exposure to environmental adversities and social inequalities, such as: (1) parents with ADHD or other mental illnesses; (2) increased exposure to risk conditions, like birth complications; (3) less access to medical care; and (4) a less stimulating family environment for the developmental process 6 . In this study, adjusting family income for gestational and perinatal factors (second and third levels) did not change the results, indicating that the effect of family income on attention and hyperactivity problems is independent of gestational risk factors and birth complications (data not shown, but available).
Children with some type of behavioral or emotional problem at 4 years showed higher odds of presenting attention and hyperactivity problems at 11 years. This suggests that such problems at 11 years were related to less specific behavioral manifestations at 4 years, an age at which the symptoms appear in less organized form, but which were detected by the mental assessment in that follow-up visit. This finding has important clinical implications, since it allows early detection of psychopathological precursors of attention and hyperactivity problems that appear at a later age, considering the difficulty in diagnosing them in preschoolers 1 . 
Table 2

Prevalence of attention and hyperactivity problems according to variables collected at 4 years of age. 1993 Pelotas (Brazil) birth cohort study, 2004-2005 follow-up.

\begin{tabular}{lcc}
\hline Variables & $\%(95 \% \mathrm{Cl})$ & p-value \\
\hline IQ & $10.5(4.1-16.8)$ & 0.009 \\
$\quad$ High & $17.3(13.3-21.2)$ & \\
$\quad$ Medium & $27.1(19.5-35.0)$ & 0.001 \\
Low & $17.1(14.1-20.3)$ & \\
Behavioral and emotional problems & $50.0(25.8-72.4)$ & 0.005 \\
$\quad$ No & $15.1(11.6-18.6)$ & \\
Yes & $26.5(19.9-33.3)$ & \\
Mother's mental disorder & & \\
No & & \\
Yes &
\end{tabular}

95\%Cl: $95 \%$ confidence interval.

Table 3

Crude and adjusted prevalence ratios (PR) with $95 \%$ confidence intervals $(95 \% \mathrm{Cl})$ for attention and hyperactivity problems according to independent variables. 1993 Pelotas (Brazil) birth cohort study, 2004-2005 follow-up.

\begin{tabular}{|c|c|c|c|c|}
\hline \multirow[t]{2}{*}{ Variables } & \multicolumn{2}{|c|}{ Crude analysis } & \multicolumn{2}{|c|}{ Adjusted analysis } \\
\hline & PR $(95 \% \mathrm{Cl})$ & p-value & PR $(95 \% \mathrm{Cl})$ & p-value \\
\hline Adolescent's gender & & $<0.001$ & & $<0.001$ \\
\hline Female & 1.00 & & 1.00 & \\
\hline Male & $1.50(1.33-1.69)$ & & $1.46(1.29-1.65)$ & \\
\hline Skin color & & 0.004 & & 0.09 \\
\hline White & 1.00 & & 1.00 & \\
\hline Black/Brown & $1.16(1.02-1.32)$ & & $1.11(0.97-1.27)$ & \\
\hline Family income (times minimum wage) & & $<0.001$ & & $<0.001$ \\
\hline$>10$ & 1.00 & & 1.00 & \\
\hline $6.1-10$ & $1.72(1.11-2.66)$ & & $1.53(1.00-2.37)$ & \\
\hline $3.1-6$ & $1.95(1.34-2.84)$ & & $1.86(1.27-2.70)$ & \\
\hline $1.1-3$ & $2.51(1.75-3.61)$ & & $2.30(1.60-3.30)$ & \\
\hline$\leq 1$ & $2.51(1.72-3.65)$ & & $2.36(1.62-3.45)$ & \\
\hline Smoking during pregnancy & & $<0.001$ & & $<0.001$ \\
\hline No & 1.00 & & 1.00 & \\
\hline Yes & $1.35(1.20-1.53)$ & & $1.28(1.13-1.45)$ & \\
\hline Alcohol during pregnancy & & 0.161 & & 0.169 \\
\hline No & 1.00 & & 1.00 & \\
\hline Yes & $1.19(0.93-1.52)$ & & $1.19(0.93-1.53)$ & \\
\hline Birth weight & & 0.361 & & \\
\hline Adequate $(\geq 2,500 \mathrm{~g})$ & 1.00 & & & \\
\hline $\operatorname{Low}(<2,500 \mathrm{~g})$ & $1.09(0.90-1.34)$ & & & \\
\hline Gestational age (weeks) & & 0.062 & & \\
\hline$\geq 37$ & 1.00 & & & \\
\hline$<37$ (premature) & $1.18(0.99-1.41)$ & & & \\
\hline Intrauterine growth restriction & & 0.129 & & \\
\hline No & 1.00 & & & \\
\hline Yes & $1.12(0.97-1.29)$ & & & \\
\hline
\end{tabular}

(continues) 


\begin{tabular}{|c|c|c|c|c|}
\hline \multirow[t]{2}{*}{ Variables } & \multicolumn{2}{|c|}{ Crude analysis } & \multicolumn{2}{|c|}{ Adjusted analysis } \\
\hline & PR (95\%Cl) & $\mathrm{p}$-value & PR $(95 \% \mathrm{Cl})$ & $\mathrm{p}$-value \\
\hline Pregnancy complications & & 0.022 & & \\
\hline No hospitalization & 1.00 & & & \\
\hline With hospitalization & $1.25(1.03-1.52)$ & & & \\
\hline Perinatal complications & & 0.100 & & \\
\hline No admission to neonatal ICU & 1.00 & & & \\
\hline With admission to neonatal ICU & $1.31(0.95-1.82)$ & & & \\
\hline 10 & & 0.003 & & 0.114 \\
\hline High & 1.00 & & 1.00 & \\
\hline Medium & $1.65(0.83-3.29)$ & & $1.42(0.71-2.83)$ & \\
\hline Low & $2.61(1.28-5.35)$ & & $1.73(0.83-3.61)$ & \\
\hline Behavioral and emotional problems & & $<0.001$ & & 0.011 \\
\hline No & 1.00 & & 1.00 & \\
\hline Yes & $2.86(1.67-4.87)$ & & $2.32(1.23-4.38)$ & \\
\hline Mother's mental disorder & & 0.003 & & 0.039 \\
\hline No & 1.00 & & 1.00 & \\
\hline Yes & $1.76(1.21-2.55)$ & & $1.50(1.02-2.21)$ & \\
\hline
\end{tabular}

Note: the effect of each variable was adjusted for the variables from the same level and higher with $p<0.20$.

Maternal mental disorder was associated with reporting of symptoms of inattention and hyperactivity in the adolescents. Since the mother's mental health assessment preceded the evaluation of the presence of ADHD symptoms, it reinforces the existence of a causal relationship between maternal mental disorder and attention and hyperactivity problems in the adolescent. Although this relationship between mental health problems in parents and attention and hyperactivity problems in children could be attributed to the increased presence of family conflict when the parents present mental illness 3,12 , it can probably be explained by genetic transmission of the risk ${ }^{3}$.

As expected, boys showed a higher risk of attention and hyperactivity problems. Neurobiological differences between the sexes makes boys more vulnerable to all early-onset mental disorders 25 , including ADHD. In addition, the symptoms of hyperactivity and impulsiveness (more frequent in boys) are more easily identified by parents and teachers than those of inattention 26 (more frequent in girls).

Maternal smoking remained associated with attention and hyperactivity problems in the adjusted analysis, even after controlling for various gestational and perinatal variables and family income, corroborating the hypothesis that smoking is an important risk factor to be considered in studies on ADHD. The association between smoking during pregnancy and externalization problems in children may be due more to the environmental risk factors associated with maternal smoking than to prenatal exposure to nicotine. Thus, maternal smoking during pregnancy is correlated with various factors besides externalization problems in children and adolescents, such as: low income, early pregnancy, lack of prenatal care, and maternal depression, among others. In addition, genetic factors can confound this association. For example, pregnant women at increased genetic risk for antisocial disorder have a higher probability of smoking 27. Although the harmful consequences of maternal smoking during pregnancy are widely known, smoking prevalence is still high. In the current study, approximately $33 \%$ of mothers smoked during pregnancy; another birth cohort study in the same city in 2004 showed that there was still a $27.5 \%$ smoking prevalence rate during pregnancy 28 , showing little change among pregnant women in relation to this habit. Considering that this risk factor can be prevented, public health strategies should focus on counseling pregnant women on the cognitive risks for their offspring resulting from smoking during pregnancy, in addition to other health hazards for both the mother and child 10 .

IQ assessed at four years did not remain associated with attention and hyperactivity problems in adolescence in the final Poisson regression model, although conceptually this association was expected 29. A possible explanation for this lack of association was the smaller size of the sample of children whose IQ was tested. Al- 
though the samples in each follow-up had different sizes, the power to detect differences was higher for the perinatal variables $(n=5,249)$ than at four years $(\mathrm{n}=634)$. In addition, WPPSI (IQ) and CBCL (behavioral and emotional problems) evaluate some similar constructs, like attention problems. According to the analysis adjusted by linear regression with attention and hyperactivity problems as a continuous variable, IQ remained associated with the outcome. The role of IQ as an independent risk factor for attention and hyperactivity problems needs to be explored in future studies with broader cognitive assessments and in the entire sample.

Black/brown skin color lost its association with attention and hyperactivity problems after controlling for family income, indicating that the increased risk of such problems is related to the effect of family income and not to race. Low birth weight was not associated with attention and hyperactivity problems in the unadjusted and adjusted analyses. Although it is widely included as a potential risk factor in the determination of attention and hyperactivity problems, various other studies have also failed to find this association 30 . Studies have suggested that this association is due more to low socioeconomic status or mental disability than to low birth weight itself 31 .

One limitation to this study was the use of a screening (rather than diagnostic) instrument for the outcome variable, although the SDQ shows high diagnostic performance.

The predictors of attention and hyperactivity problems identified in this study have been widely discussed in the literature as probable risk factors for ADHD. However, our results were obtained with a population-based study and the information was collected prospectively, unlike the previous findings, mostly from studies with clinical samples including a smaller number of subjects and data collected retrospectively. The results confirm the importance of early-life events for attention and hyperactivity problems in adolescence. Comparison with findings from studies performed in various countries 4,9,12,30 showed the similarity of risk factors for attention and hyperactivity problems in different cultural contexts.

\section{Resumo}

O objetivo deste trabalho foi estudar determinantes precoces dos problemas de atenção e hiperatividade em adolescentes. Em 1993, os nascimentos foram recrutados e as mães entrevistadas $(n=5.249)$. Aos 11 anos, 4.423 mães responderam ao Strengths and Difficulties Questionnaire (SDQ) para avaliar problemas de atenção e hiperatividade nos adolescentes. Variáveis socioeconômicas, demográficas, biológicas e psicológicas foram investigadas como prováveis fatores de risco. Razões de prevalência brutas e ajustadas foram calculadas com regressão de Poisson. A prevalência de problemas de atenção e hiperatividade foi 19,9\%. Sexo masculino, baixa renda familiar, tabagismo materno na gestação, transtornos psiquiátricos maternos e problemas de comportamento/emocionais do adolescente aos quatro anos permaneceram associados na análise ajustada. Eventos do início da vida influenciaram os problemas de atenção e hiperatividade na adoles cência. Os fatores de risco para problemas de atenção e hiperatividade foram similares aos encontrados em diferentes culturas.

Transtorno do Déficit de Atenção com Hiperatividade; Adolescente; Estudos de Coorte 


\section{Contributors}

L. Anselmi designed the research project, reviewed the instruments, participated in the data collection, analyzed the data, and wrote the article. A. M. B. Menezes, F. C. Barros, P. C. Hallal, and C. L. Araújo participated in the study design and planning, data collection, and revision of the article. M. R. Domingues collaborated in the statistical analysis and writing the article. L. A Rohde designed the research project, analyzed the data, and wrote the article.

\section{Acknowledgments}

The cohort study is supported by the Wellcome Trust. The initial phases of the cohort were funded by the European Union and the Brazilian National Program for Centers of Excellence (PRONEX), National Research Council (CNPq), and Ministry of Health. Luciana Anselmi received a $\mathrm{PhD}$ scholarship from the Coordinating Division for Graduate Studies (CAPES) through the Program for Graduate Studies in Psychiatry, Federal University in Rio Grande do Sul (UFRGS). The authors thank Prof. Bernardo Horta (Federal University in Pelotas - UFPel) for his revision of the statistical analysis.

\section{References}

1. American Psychiatric Association. Diagnostic and statistical manual of mental disorders, DSM-IV. $4^{\text {th }}$ Ed. Washington DC: American Psychiatric Association; 1994.

2. Leibson CL, Long KH. Economic implications of attention-deficit hyperactivity disorder for healthcare systems. Pharmacoeconomics 2003; 21:1239-62.

3. Biederman J, Faraone SV. Attention-deficit hyperactivity disorder. Lancet 2005; 366:237-48.

4. Pineda DA, Palacio LG, Puerta IC, Merchán V, Arango CP, Galvis AY, et al. Environmental influences that affect attention deficit/hyperactivity disorder: study of a genetic isolate. Eur Child Adolesc Psychiatry 2007; 16:337-46.

5. Costello EJ, Angold A, Burns BJ, Stangl DK, Tweed DL, Erkanli A, et al. The Great Smoky Mountains Study of Youth. Goals, design, methods, and the prevalence of DSM-III-R disorders. Arch Gen Psychiatry 1996; 53:1129-36.

6. Pineda D, Ardila A, Rosselli M, Arias BE, Henao GC, Gomez LF, et al. Prevalence of attention-deficit/ hyperactivity disorder symptoms in 4- to 17-yearold children in the general population. J Abnorm Child Psychol 1999; 27:455-62.

7. Rhee SH, Waldman ID, Hay DA, Levy F. Sex differences in genetic and environmental influences on DSM-III-R attention-deficit/hyperactivity disorder. J Abnorm Psychol 1999; 108:24-41.

8. Hillemeier MM, Foster EM, Heinrichs B, Heier B; Conduct Problems Prevention Research Group. Racial differences in parental reports of attentiondeficit/hyperactivity disorder behaviors. J Dev Behav Pediatr 2007; 28:353-61.

9. Kotimaa AJ, Moilanen I, Taanila A, Ebeling H, Smalley SL, McGough JJ, et al. Maternal smoking and hyperactivity in 8-year-old children. J Am Acad Child Adolesc Psychiatry 2003; 42:826-33.

10. Schmitz M, Denardin D, Laufer Silva T, Pianca T, Hutz MH, Faraone S, et al. Smoking during pregnancy and attention-deficit/hyperactivity disorder, predominantly inattentive type: a case-control study. J Am Acad Child Adolesc Psychiatry 2006; 45:1338-45.

11. Winzer-Serhan UH. Long-term consequences of maternal smoking and developmental chronic nicotine exposure. Front Biosci 2008; 13:636-49.

12. Pressman LJ, Loo SK, Carpenter EM, Asarnow JR, Lynn D, McCracken JT, et al. Relationship of family environment and parental psychiatric diagnosis to impairment in ADHD. J Am Acad Child Adolesc Psychiatry 2006; 45:346-54.

13. Araújo CL, Menezes AMB, Vieira MFA, Neutzling MB, Gonçalves H, Anselmi L, et al. The 11-year follow-up of the 1993 Pelotas (Brazil) birth cohort study: methods. Cad Saúde Pública 2010; 26: 1875-86. 
14. Dubowitz LM, Dubowitz V, Goldberg C. Clinical assessment of gestational age in the newborn infant. J Pediatr 1970; 77:1-10.

15. Achenbach TM. Manual for the Child Behavior Checklist/4-18 and 1991 profile. Burlington: University of Vermont; 1991.

16. Wechsler D. Test de Inteligencia para Preescolares (WPPSI) manual. Buenos Aires: Paidós; 1991.

17. Mari JJ, Williams P. A validity study of a psychiatric screening questionnaire (SRQ-20) in primary care in the city of São Paulo. Br J Psychiatry 1986; 148:23-6.

18. Goodman R. Psychometric properties of the strengths and difficulties questionnaire. J Am Acad Child Adolesc Psychiatry 2001; 40:1337-45.

19. Rohde LA, Biederman J, Knijnik M, Ketzer C, Chachamovich E, Vieira GM, et al. Exploring different information sources for DSM-1V ADHD diagnoses in Brazilian adolescents. J Atten Disord 1999; 3:91-6.

20. Fleitlich-Bilyk B, Goodman R. Prevalence of child and adolescent psychiatric disorders in southeast Brazil. J Am Acad Child Adolesc Psychiatry 2004; 43:727-34.

21. Bordin I, Mari J, Caeiro M. Validação da versão brasileira do "Child Behavioral Checklist" (CBCL) (Inventário de Comportamentos da Infância e Adolescência): dados preliminares. Rev Bras Psiquiatr 1995; 17:55-66.

22. Harding TW, Arango MV, Baltazar J, Climent CE, Ibrahim HH, Ladrido-Ignacio L, et al. Mental disorders in primary health care: a study of their frequency and diagnosis in four developing countries. Psychol Med 1980; 10:231-41.

23. Barros AJ, Hirakata VN. Alternatives for logistic regression in cross-sectional studies: an empirical comparison of models that directly estimate the prevalence ratio. BMC Med Res Methodol 2003; $3: 21$.
24. Victora CG, Huttly SR, Fuchs SC, Olinto MT. The role of conceptual frameworks in epidemiological analysis: a hierarchical approach. Int J Epidemiol 1997; 26:224-7.

25. Rutter M, Caspi A, Moffitt TE. Using sex differences in psychopathology to study causal mechanisms: unifying issues and research strategies. J Child Psychol Psychiatry 2003; 44:1092-115.

26. Staller J, Faraone SV. Attention-deficit hyperactivity disorder in girls: epidemiology and management. CNS Drugs 2006; 20:107-23.

27. D'Onofrio BM, Van Hulle CA, Waldman ID, Rodgers JL, Harden KP, Rathouz PJ, et al. Smoking during pregnancy and offspring externalizing problems: an exploration of genetic and environmental confounds. Dev Psychopathol 2008; 20:139-64.

28. Domingues MR, Barros AJ. Leisure-time physical activity during pregnancy in the 2004 Pelotas Birth Cohort Study. Rev Saúde Pública 2007; 41:173-80.

29. Koenen KC, Moffitt TE, Roberts AL, Martin LT, Kubzansky L, Harrington $\mathrm{H}$, et al. Childhood IQ and adult mental disorders: a test of the cognitive reserve hypothesis. Am J Psychiatry 2009; 166:50-7.

30. Sato M, Aotani H, Hattori R, Funato M. Behavioral outcome including attention deficit/hyperactivity disorder and minor neurological signs in perinatal high-risk newborns at 4-6 years of age with relation to risk factors. Pediatr Int 2004; 46:346-52.

31. Kreppner JM, O'Connor TG, Rutter M. Can inattention/overactivity be an institutional deprivation syndrome? J Abnorm Child Psychol 2001; 29: 513-28.

Submitted on $15 / \mathrm{Feb} / 2009$

Final version resubmitted on 01/Sep/2009

Approved on 14/Oct/2009 